PROCEEDINGS OF THE

AMERICAN MATHEMATICAL SOCIETY

Volume 130, Number 4 , Pages 1073-1078

S 0002-9939(01)06265-7

Article electronically published on September 14, 2001

\title{
ACL HOMEOMORPHISMS AND LINEAR DILATATION
}

\author{
S. KALLUNKI AND O. MARTIO \\ (Communicated by Juha M. Heinonen)
}

\begin{abstract}
We establish an integrability condition on the linear dilatation to guarantee ACL.
\end{abstract}

\section{INTRODUCTION}

Let $D$ be a domain in $\mathbf{R}^{n}, n \geq 1$, and $f: D \rightarrow \mathbf{R}^{n}$ a homeomorphism. For $x \in D$ and $0<r<d(x, \partial D)$ we set

$$
\begin{gathered}
L(x, f, r)=\sup \{|f(x)-f(y)|: y \in \partial B(x, r)\}, \\
l(x, f, r)=\inf \{|f(x)-f(y)|: y \in \partial B(x, r)\}
\end{gathered}
$$

where $B(x, r)$ stands for the open ball centered at $x$ and radius $r$ and $\partial B(x, r)$ for its boundary. The linear dilatation of $f$ at $x$ is defined as

$$
H(x, f)=\limsup _{r \rightarrow 0} H(x, f, r)
$$

where $H(x, f, r)=L(x, f, r) / l(x, f, r)$. At every point $x \in D, H(x, f) \in[1, \infty]$ and $H(x, f)=\left\|f^{\prime}(x)\right\| / l\left(f^{\prime}(x)\right)$ provided that $f$ is differentiable at $x$ with $l\left(f^{\prime}(x)\right)>0$. Here the norm $\left\|f^{\prime}(x)\right\|$ of the derivative $f^{\prime}(x)$ of $f$ at $x$ is defined as

$$
\left\|f^{\prime}(x)\right\|=\sup _{|h|=1}\left|f^{\prime}(x) h\right|
$$

and the minimum norm $l\left(f^{\prime}(x)\right)$ as

$$
l\left(f^{\prime}(x)\right)=\inf _{|h|=1}\left|f^{\prime}(x) h\right| .
$$

A well-known result of Gehring [G1] says that if a homeomorphism $f$ has its linear dilatation $H(x, f)$ uniformly bounded in $D$, then $f$ is a quasiconformal mapping. In particular $f$ is ACL in $D$, which means that $f$ is absolutely continuous on almost every line segment parallel to the coordinate axis in $D$. In [T2] Tukia conjectured that the condition

$$
m(\{x \in D: H(x, f)>t\})<c t^{-\alpha}
$$

for some $\alpha>3$ is sufficient for the ACL property of a plane homeomorphism $f$. Indeed, this was proved in $[\mathrm{FA}$ together with a space analogue. In this paper we show that $\alpha>2$ in (1.1) implies the ACL property in the plane case with a

Received by the editors October 5, 2000

2000 Mathematics Subject Classification. Primary 30C62, 30 C65.

The first author was partially supported by the foundation Vilho, Yrjö ja Kalle Väisälän rahasto. 
corresponding improvement in space. Our proof is based on the Gehring method in G1.

Theorem 1.2. Suppose that a homeomorphism $f: D \rightarrow \mathbf{R}^{n}, D \subset \mathbf{R}^{n}$ a domain, a subset $S$ of $D$ and $s \in(1, \infty]$ satisfy the conditions:

(1.3) $s>n /(n-1)$,

(1.4) $H(x, f)<\infty$ for each $x \in D \backslash S$,

(1.5) $H(x, f) \in L_{l o c}^{s}(D)$,

(1.6) $S$ has $\sigma$-finite $(n-1)$-Hausdorff measure.

Then $f$ is $A C L$ in $D$.

Remarks 1.7. (a) The assumption (1.3) rules out the case $n=1$, see Section 2 .

(b) The assumption (1.6) means that the set $S$ is of the form $S=\bigcup S_{i}$ where $H^{n-1}\left(S_{i}\right)<\infty$ and $H^{n-1}$ refers to the $(n-1)$-dimensional Hausdorff measure. For the definition of the Hausdorff measure see e.g. [V] p. 99] or [G1].

(c) We do not know the optimal value for $s$ in Theorem 1.2 An example shows that one cannot take $s<1$. In this example the positive real axis, and lines parallel to it, are mapped to the curve $x \sin (1 / x)$ and to its translations. This construction produces a non-ACL homeomorphism $f$ of $\mathbf{R}^{2}$ which is the identity on the left half plane and satisfies $H(x, f) \in L_{l o c}^{s}\left(\mathbf{R}^{2}\right)$ for any $s<1$.

In Section 2 we consider some properties of homeomorphisms $f: D \rightarrow \mathbf{R}^{n}$ satisfying $H(x, f)<\infty$ a.e. in $D$. In Corollary 2.4 we show that $f^{\prime} \in L_{l o c}^{p}(D)$, $p=s n /(n-1+s)$, under the conditions of Theorem 1.2 In particular this implies that $f$ is $\mathrm{ACL}^{p}$. The section also contains some examples. Section 3 is devoted to the proof of Theorem 1.2.

\section{Mappings with $H(x, f)<\infty$ A.E.}

If a homeomorphism $f: D \rightarrow \mathbf{R}^{n}$ satisfies $H(x, f)<\infty$ for a.e. $x \in D$ or

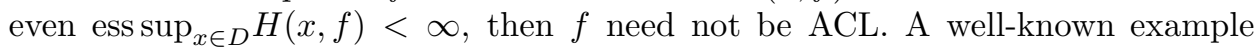
is constructed from the Cantor staircase function $g:[0,1] \rightarrow[0,1]$, i.e. $g$ is an increasing function with the property $g^{\prime}(x)=0$ for a.e. $x \in[0,1]$. Now $f:(0,1) \times$ $(0,1) \rightarrow(0,2) \times(0,1)$ defined as $f(x, y)=(g(x)+x, y)$ is a homeomorphism with $H(z, f)=1$ a.e. but $f$ is not ACL. Moreover, no boundedness condition, except $H(x, f)=1$ for all $x$, in the case $n=1$ implies absolute continuity. Indeed, an increasing homeomorphism $f: \mathbf{R} \rightarrow \mathbf{R}$ is called $K$-quasisymmetric if it satisfies

$$
\frac{1}{K} \leq \frac{f(x+t)-f(x)}{f(x)-f(x-t)} \leq K
$$

for all $x \in \mathbf{R}$ and $t>0$. If $f$ is $K$-quasisymmetric, then $H(x, f) \leq K$ for all $x \in \mathbf{R}$. Beurling and Ahlfors $[\mathrm{BA}]$ constructed for each $K>1$ a $K$-quasisymmetric mapping $f$ which is not absolutely continuous. For more striking examples of such mappings see [T1]. Hence no integrability condition for $H(x, f)$ like $(1.5)$ implies absolute continuity for $n=1$.

However, homeomorphisms which satisfy $H(x, f)<\infty$ a.e. have some nice properties.

Theorem 2.1. Suppose that a homeomorphism $f: D \rightarrow \mathbf{R}^{n}$ satisfies $H(x, f)<\infty$ a.e. in D. Then $f$ is a.e. differentiable. 
Proof. Fix an open set $G \subset \subset D$ and let $\Phi(E)=|f(E)|$ for each Borel set $E \subset G$. Then $\Phi$ is a finite Borel measure on $G$ and hence it has a finite derivative

$$
\Phi^{\prime}(x)=\lim _{r \rightarrow 0} \frac{\Phi(B(x, r))}{|B(x, r)|}
$$

at a.e. $x \in G$. Here and in the following $|A|$ means the Lebesgue measure of a set $A \subset \mathbf{R}^{n}$.

Now at almost every point $x$ of $G, \Phi^{\prime}(x)$ exists and $H(x, f)<\infty$. Fix such a point $x$. Let $y \in G$ with $0<|x-y|<d(x, \partial G)$. Now

$$
\begin{aligned}
\left(\frac{|f(x)-f(y)|}{|y-x|}\right)^{n} & \leq\left(\frac{L(x, f,|y-x|)}{l(x, f,|y-x|)}\right)^{n}\left(\frac{l(x, f,|y-x|)}{|y-x|}\right)^{n} \\
& \leq H(x, f,|y-x|)^{n} \frac{\Phi(B(x,|y-x|))}{|B(x,|y-x|)|}
\end{aligned}
$$

and letting $y \rightarrow x$ we see that

$$
\limsup _{y \rightarrow x} \frac{|f(y)-f(x)|}{|y-x|} \leq H(x, f) \Phi^{\prime}(x)^{\frac{1}{n}}<\infty .
$$

By the Rademacher-Stepanov theorem the mapping $f$ is a.e. differentiable in $G$. The theorem follows.

Theorem 2.2. Suppose that a homeomorphism $f: D \rightarrow \mathbf{R}^{n}$ satisfies $H(x, f) \in$ $L_{l o c}^{s}(D), s \in[1, \infty]$. Then $f^{\prime} \in L_{l o c}^{p}(D)$ with $p=s n /(n-1+s)$ and $p=n$ if $s=\infty$.

Proof. We may assume that $f$ is sense-preserving. Since $H(x, f)<\infty$ a.e. in $D$, Theorem 2.1 implies that $f^{\prime}(x)$ exists a.e. If $f$ is differentiable at $x$ and $H(x, f)<$ $\infty$, then an elementary argument shows that

$$
\left\|f^{\prime}(x)\right\|^{n} \leq H(x, f)^{n-1} J(x, f)
$$

where $J(x, f)$ is the Jacobian determinant of $f^{\prime}(x)$.

Fix an open set $G \subset \subset D$. For $s<\infty(\underline{2.3})$ and the Hölder inequality imply

$$
\begin{aligned}
\int_{G}\left|f^{\prime}(x)\right|^{p} d x & \leq \int_{G} H(x, f)^{\frac{p(n-1)}{n}} J(x, f)^{\frac{p}{n}} d x \\
& \leq\left[\int_{G} H(x, f)^{\frac{p(n-1)}{(n-p)}} d x\right]^{\frac{(n-p)}{n}}\left[\int_{G} J(x, f) d x\right]^{\frac{p}{n}} \\
& \leq\left[\int_{G} H(x, f)^{s} d x\right]^{\frac{(n-p)}{n}}|f(G)|^{\frac{p}{n}}<\infty
\end{aligned}
$$

as required. For $s=\infty$ the proof is similar. Note that the inequality

$$
\int_{G} J(x, f) d x \leq|f(G)|
$$

always holds for an a.e. differentiable homeomorphism; see [RR, p. 360].

Corollary 2.4. Under the hypotheses of Theorem $1.2 f$ is a.e. differentiable and $f^{\prime} \in L_{l o c}^{p}(D), p=s n /(n-1+s)$. In particular $f$ is $A C L^{p}$ in $D$. 


\section{Proof of Theorem 1.2}

We prove Theorem 1.2 in the case $S=\emptyset$. By the theorem of Gross (see e.g. V p. 103]) the condition (111.2) implies that for a.e. line $L$ parallel to some coordinate axis, $S \cap L$ is countable. For a continuous function a countable set $E$ does not destroy absolute continuity if an estimate like (3.8) below holds for compact sets $F$ in the complement of $E$. Thus the case $S \neq \emptyset$ does not lead to essential difficulties; see [G1].

Pick a closed cube $Q \subset \subset D$ whose sides are parallel to the coordinate axes and write $Q^{\prime}=\frac{1}{2} Q$ for the cube with the same center as $Q$ and side length half of that of $Q$. In order to show that $f$ is ACL it suffices to show that $f$ is absolutely continuous on almost every line segment of $Q^{\prime}$ parallel to the coordinate axes. Renormalizing we may assume that $Q=[-2,2]^{n}$ and by symmetry it is sufficient to consider segments parallel to the $x_{n}$-axis. Let $P: \mathbf{R}^{n} \rightarrow \mathbf{R}^{n-1}$ denote the projection $P(x)=x-x \cdot e_{n} e_{n}$ and for $y \in P(Q) \subset \mathbf{R}^{n-1}$ write $I=I(y)=Q^{\prime} \cap P^{-1}(y)$ for the line segment parallel to the $x_{n}$-axis in $Q^{\prime}$.

Next for a Borel set $E \subset P(Q)$ write

$$
\Phi(E)=\left|f\left(Q \cap P^{-1}(E)\right)\right| \leq|f(Q)|<\infty .
$$

Then $\Phi$ is a finite Borel measure on $P(Q)$ and hence it has a finite derivative $\Phi^{\prime}(y)$ for almost all $y \in P\left(Q^{\prime}\right)$. We choose $y \in P\left(Q^{\prime}\right)$ such that (i) $\Phi^{\prime}(y)$ exists and (ii) $H(x, f) \in L^{s}(I(y))$. The last assertion follows from the Fubini theorem. It suffices to show that $f$ is absolutely continuous on $I(y)$.

To this end let $F \subset I(y)$ be a compact set. For each $k=0,1,2, \ldots$ write

$$
F_{k}=\left\{x \in F: 2^{k} \leq H(x, f)<2^{k+1}\right\} .
$$

Then $F_{k}$ is a Borel set and $F=\bigcup F_{k}$ because of (1.4) and our assumption $S \neq \emptyset$. Note also that $H(x, f) \geq 1$ for every $x$. We first derive the estimate

$$
H^{1}\left(f F_{k}\right) \leq C 2^{k} H^{1}\left(F_{k}\right)^{\frac{n-1}{n}}
$$

where $C=\left(2^{2 n+1} \Phi^{\prime}(y)\right)^{1 / n}$.

For (3.1) fix $k$ and for each $j=1,2, \ldots$ consider the set

$$
F_{k, j}=\left\{x \in F_{k}: L(x, f, r)^{n} \leq 2^{n(k+1)}|f B(x, r)| / \Omega_{n} \text { for } 0<r<1 / j\right\}
$$

where $\Omega_{n}=|B(0,1)|$. The sets $F_{k, j}$ are Borel sets and $F_{k, j} \subset F_{k, j+1}$ with

$$
F_{k}=\bigcup_{j=1}^{\infty} F_{k, j} .
$$

To see (3.2) let $x \in F_{k}$. Then $H(x, f)<2^{k+1}$ and hence there is $j$ such that

$$
L(x, f, r) / l(x, f, r)<2^{k+1}
$$

for all $0<r<1 / j$ and we obtain

$$
L(x, f, r)^{n}<2^{n(k+1)} l(x, f, r)^{n} \leq 2^{n(k+1)}|f B(x, r)| / \Omega_{n} .
$$

This shows that $x \in F_{k, j}$ and (3.2) follows.

By the monotonicity and (3.2) it suffices to prove (3.1) for $F_{k, j}$ instead of $F_{k}$. Fix $j$ and let $F^{\prime}$ be an arbitrary compact subset of $F_{k, j}$. Let $\epsilon>0$ and $t>0$. The continuity of the mapping $(x, r) \mapsto L(x, f, r)$ gives $\delta, 0<\delta<1 / j$, such that $L(x, f, r)<t / 2$ for $0<r<\delta$ and for all $x \in F^{\prime}$. By a well-known covering lemma for sets on a real line (see [G1, Lemma 1, p. 6]), for each sufficiently small 
$r>0,0<r<\delta$, there exists a covering of $F^{\prime}$ by a finite number of open balls $B_{i}=B\left(x_{i}, r\right), i=1, \ldots, l$, where (a) $x_{i} \in F^{\prime}, i=1, \ldots, l$, (b) each point of $\mathbf{R}^{n}$ lies in at most two $B_{i}$ and (c) $l r \leq H^{1}\left(F^{\prime}\right)+\epsilon$. Note that the normalizing condition gives

$$
B_{i} \subset Q \cap P^{-1}(B)
$$

where $B=B^{n-1}(y, r)$.

The union of the sets $f\left(B_{i}\right)$ covers $f\left(F^{\prime}\right)$ and

$$
\operatorname{dia}\left(f B_{i}\right) \leq 2 L\left(x_{i}, f, r\right)<t .
$$

Hence

$$
H_{t}^{1}\left(f F^{\prime}\right) \leq \sum_{i=1}^{l} \operatorname{dia}\left(f B_{i}\right)
$$

where

$$
H_{t}^{1}(A)=\inf \left\{\sum \operatorname{dia}\left(A_{i}\right): \bigcup A_{i} \supset A, \operatorname{dia}\left(A_{i}\right)<t\right\}
$$

and the Hölder inequality together with the definition of $F_{k, j}$ yields

$$
\begin{aligned}
H_{t}^{1}\left(f F^{\prime}\right)^{n} & \leq\left(\sum_{i=1}^{l} \operatorname{dia}\left(f B_{i}\right)\right)^{n} \leq l^{n-1} \sum_{i=1}^{l} \operatorname{dia}\left(f B_{i}\right)^{n} \\
& \leq l^{n-1} 2^{n} \sum_{i=1}^{l} L\left(x_{i}, f, r\right)^{n} \leq \frac{l^{n-1} 2^{n} 2^{n(k+1)}}{\Omega_{n}} \sum_{i=1}^{l}\left|f B_{i}\right| .
\end{aligned}
$$

Since $f$ is a homeomorphism, we obtain from (b) and (3.3) that

$$
\sum_{i=1}^{l}\left|f B_{i}\right| \leq 2\left|\bigcup_{i=1}^{l} f B_{i}\right| \leq 2 \Phi(B)
$$

and thus (3.4) and (c) yield

$$
\begin{aligned}
H_{t}^{1}\left(f F^{\prime}\right)^{n} & \leq 2^{n(k+2)+1}\left(H^{1}\left(F^{\prime}\right)+\epsilon\right)^{n-1} \Phi(B) / H^{n-1}(B) \\
& \leq 2^{n(k+2)+1}\left(H^{1}\left(F_{k, j}\right)+\epsilon\right)^{n-1} \Phi(B) / H^{n-1}(B) .
\end{aligned}
$$

Since $H_{t}^{1}\left(f F^{\prime}\right) \rightarrow H^{1}\left(f F^{\prime}\right)$ as $t \rightarrow 0$, letting first $r \rightarrow 0$, then $\epsilon \rightarrow 0$, and finally $t \rightarrow 0$ we obtain

$$
H^{1}\left(f F^{\prime}\right)^{n} \leq 2^{n(k+2)+1} H^{1}\left(F_{k, j}\right)^{n-1} \Phi^{\prime}(y) .
$$

Now $F^{\prime}$ is an arbitrary compact subset of $F_{k, j}$ and hence (3.5) holds for $F_{k, j}$ on the left-hand side of (3.5). This leads to the estimate (3.1).

Since $f F=\bigcup f F_{k}$, (3.1) implies

$$
H^{1}(f F) \leq \sum H^{1}\left(f F_{k}\right) \leq C \sum 2^{k} H^{1}\left(F_{k}\right)^{\frac{n-1}{n}} .
$$

The sets $F_{k}, k=1,2, \ldots$, are disjoint and hence the integral estimate

$$
\sum_{k=0}^{\infty} 2^{k s} H^{1}\left(F_{k}\right) \leq \int_{F} H(x, f)^{s} d x_{n}
$$


is elementary. From (3.6), (3.7) and from the Hölder inequality we obtain

$$
\begin{aligned}
H^{1}(f F) & \leq C_{1}\left(\sum_{k=0}^{\infty} 2^{k s} H^{1}\left(F_{k}\right)\right)^{\frac{n-1}{n}}\left(\sum_{k=0}^{\infty} 2^{k(n-s(n-1))}\right)^{\frac{1}{n}} \\
& \leq C_{2}\left(\int_{F} H(x, f)^{s} d x_{n}\right)^{\frac{n-1}{n}}
\end{aligned}
$$

where $C_{2}$ depends only on $n, s$ and $\Phi^{\prime}(y)$. Note that the series

$$
\sum_{k=0}^{\infty} 2^{k(n-s(n-1))}
$$

converges because $s>n /(n-1)$ and hence $n-s(n-1)<0$. Inequality (3.8) shows that $f$ is absolutely continuous on $I(y)$ as required.

\section{REFERENCES}

[BA] Beurling, A. and Ahlfors, L.V., The boundary correspondence under quasiconformal mappings, Acta Math. 96 (1956), 125-142. MR 19:258c

[FA] Fang Ainong, The ACL property of homeomorphisms under weak conditions, Acta Math. Sin., New Series 14.4 (1998), 473-480. MR 2000e:30035

[G1] Gehring, F.W., The definitions and exceptional sets for quasiconformal mappings, Ann. Acad. Sci. Fenn. Ser. AI 281 (1960), 1-28. MR 23:A1800

[G2] Gehring, F.W., Lower dimensional absolute continuity properties of quasiconformal mappings, Math. Proc. Camb. Phil. Soc. 78 (1975), 81-92. MR 52:3520

[RR] Rado, T. and Reichelderfer, P.V., Continuous transformations in analysis, Die Grundlehren der matematischen Wissenschaften 75, Springer-Verlag, 1955. MR 18:115c

[T1] Tukia, P., Hausdorff dimension and quasisymmetric mappings, Math. Scand. 65 (1989), 152-160. MR 92b:30026

[T2] Tukia, P., Compactness properties of $\mu$-homeomorphisms, Ann. Acad. Sci. Fenn. Ser. AI Math. 16 (1991), 47-69. MR 93c:30029]

[V] Väisälä, J., Lectures on n-dimensional quasiconformal mappings, Lecture Notes in Mathematics 229. Springer-Verlag, 1971. MR 56:12260

Department of Mathematics, University of Jyvëskylä, P.O. Box 35, Fin-40351 JYVÄSKYLÄ, FINLAND

E-mail address: sakallun@maths.jyu.fi

Department of Mathematics, P.O. Box 4, Fin-00014 University of Helsinki, Finland

E-mail address: olli.martio@helsinki.fi 